\title{
Use of a breeding bull and absence of a calving pen as risk factors for the presence of Mycoplasma bovis in dairy herds
}

\author{
Linde Gille, ${ }^{* 1}$ J. Callens, † K. Supré,‡ F. Boyen,§ F. Haesebrouck,§ L. Van Driessche, ${ }^{*}$ K. van Leenen, ${ }^{*}$ \\ P. Deprez, ${ }^{*}$ and B. Pardon* \\ *Department of Large Animal Internal Medicine, Faculty of Veterinary Medicine, Ghent University, Ghent, Belgium, 9820 \\ †Animal Health Service Flanders (DGZ Vlaanderen), Torhout, Belgium, 8820 \\ łFlanders Milk Control Centre (MCC Vlaanderen), Lier, Belgium, 2500 \\ $\S$ Department of Pathology, Bacteriology and Avian Disease, Faculty of Veterinary Medicine, Ghent University, Ghent, Belgium, 9820
}

\begin{abstract}
Mycoplasma bovis is an important cause of pneumonia and mastitis in cattle throughout the world, often reported as emerging. In absence of an effective vaccine for $M$. bovis, current prevention and control strategies rely on the identification of risk factors for within- and between-herd spread. The objective of this study was to determine the prevalence of $M$. bovis in Belgian dairy herds and to identify risk factors associated with a positive PCR or antibody ELISA bulk tank milk (BTM) test. A cross-sectional study was performed in 2016 on 100 dairy farms, analyzing BTM using PCR and antibody ELISA. Information on herd-level risk factors focusing on biosecurity and management were collected through a questionnaire and sourced from the national herd identification system (SANITRACE, Animal Health Service Flanders). Multivariable logistic regression was used to identify herd-level risk factors for the presence of $M$. bovis DNA and antibodies in BTM. The apparent prevalence on BTM was 7 and $17 \%$ for PCR and antibody ELISA, respectively. The true prevalence was $7.1 \%$ [95\% confidence interval $(\mathrm{CI})=2.1-11.5 \%$ ] and $24.8 \%(95 \% \mathrm{CI}=16.4-33.2 \%)$. There was no overlap between ELISA- and PCR-positive farms, resulting in a combined true prevalence of $31.8 \%$ of the Belgian farms being in recent contact with $M$. bovis. Risk factor analysis showed that herds with a breeding bull $[M$. bovis-positive results for 45.5 and $13.6 \%$ of herds with and without a bull, respectively, odds ratio $=4.7(95 \%$ $\mathrm{CI}=1.1-19.8)]$ and without a calving pen $[M$. bovispositive result in 52.4 and $20.6 \%$ of the herds without and with a calving pen, respectively, odds ratio $=3.7$ $(95 \% \mathrm{CI}=1.06-12.5)]$ had higher odds to harbor $M$. bovis antigen or antibodies in BTM. In conclusion, the
\end{abstract}

Received April 18, 2018

Accepted May 26, 2018

${ }^{1}$ Corresponding author: Linde.gille@ugent.be present study points to a several fold increase in the prevalence of $M$. bovis in Belgian dairy herds. The importance of the breeding bull and calving pen in the between- and within-herd spread of $M$. bovis might have been underestimated in the past. Focusing on these factors might contribute to more effective control programs in the future.

Key words: biosecurity, bulk tank milk, control, prevalence

\section{INTRODUCTION}

Mycoplasma bovis is the Mycoplasma species with the highest economic impact in cattle (Nicholas and Ayling, 2003). Highly contagious, it can cause a variety of clinical presentations, of which (chronic) pneumonia, arthritis and otitis in calves and mastitis and pneumonia in adult cattle are the most common (Maunsell et al., 2011).

Historically, only culture has been available to assess the prevalence of $M$. bovis (Sachse et al., 1993). The development of commercial antibody (Ab) ELISA and PCR tests has facilitated further $M$. bovis screening in animals. Antibody ELISA and PCR have been validated for use on bulk tank milk (BTM) and composite milk samples, serving as an easy-to-use indicator for the presence of M. bovis in a herd. (Cai et al., 2005; Nielsen et al., 2015; Parker et al., 2017). The sensitivity and specificity of a commercially available ELISA (Bio-X BIO K 302, Bio-X Diagnostics, Rochefort, Belgium) on BTM are estimated to be $60.4 \%(95 \% \mathrm{CI}=37.5-96.2)$ and $97.3 \%$ (95\% CI $=94-99.8)$, respectively (Nielsen et al., 2015). Approximately 30\% of the lactating animals in a herd need to produce antibodies against $M$. bovis before the BTM sample will test positive on ELISA (Petersen et al., 2016). After a clinical outbreak, the antibodies stay present in BTM for about 8 mo (Parker et al., 2017). The PCR has a higher sensitivity, but relies on the active excretion of $M$. bovis (Sachse et al., 2010). As M. bovis may be shed intermittently, and the 
milk of mastitic cows is supposed to be withheld from the BTM, this could lead to an underestimation of the $M$. bovis herd prevalence when relying solely on a single PCR analysis (Petersen et al., 2016).

Current approaches for $M$. bovis control are highly variable and based on the available knowledge of $M$. bovis spread (Maunsell et al., 2011). Between herds, the biggest $M$. bovis introduction risk is attributed to the purchase of carrier animals. Between adult animals, the milking process and direct contact are seen as the main causes of transmission (Maunsell et al., 2011). In calves, feeding of milk from infected cows (such as feeding waste milk) is seen as an important cause (Maunsell and Donovan, 2009). Other causes of transmission, such as via fomites, airborne or aerosol spreading, and colostrum, have been suggested (Godden et al., 2006; Gille et al., 2016; Calcutt et al., 2018). Recently, infected semen was linked to the introduction of $M$. bovis into 2 closed herds (Haapala et al., 2018).

Elimination of $M$. bovis is very difficult to impossible. Especially in cases of $M$. bovis mastitis, experts urge separation and culling of affected cattle instead of attempting treatment (González and Wilson, 2003; Fox et al., 2005; Nicholas et al., 2016). As treatment options are limited, prevention on the herd and cattle levels alike is key. Given the current lack of a useable vaccine (Perez-Casal et al., 2017), prevention hinges on the identification and elimination of epidemiological risk factors for $M$. bovis infection. Unfortunately, only a limited number of risk factors have been identified to this date. Having a larger herd size showed a strong association (15 times higher odds) with having mycoplasma in the BTM (Thomas et al., 1981). In the same study, a small association was also observed between a high culling rate and a positive BTM was seen. Gonzalez et al. (1992), however, could not find an association between herd size and mycoplasmal mastitis. A study by Burnens et al. (1999) found only the purchase of animals was significantly linked to the serological M. bovis status of a herd (odds ratio $=10.8$ ). Aebi et al. (2015) found that farms applying forestripping, having a high average milk production or many herd movements, had higher odds of having $M$. bovis present. The objectives of our study were to investigate the prevalence of $M$. bovis in dairy herds in Belgium by determining the presence of $M$. bovis DNA and antibodies in BTM samples and to identify risk factors for a positive BTM sample.

\section{MATERIALS AND METHODS}

A cross-sectional study was conducted on 100 dairy herds in Flanders (Belgium) in January 2016. The study population was randomly selected (aselect procedure in Microsoft Excel 2016, Microsoft Corp., Redmond, WA) from the national cattle identification database (SANITRACE, Animal Health Service Flanders). The herd selection was stratified on province according to cattle density. Sample size calculation was done through WIN EPISCOPE (Win Episcope 2.0, Zaragoza, Spain). A sample size of 97 herds was calculated from the pool of approximately 6,600 Flemish dairy herds (Flemish Department of Agriculture and Fisheries, 2016) using a worst case $M$. bovis prevalence of $50 \%$, with $80 \%$ power and $95 \%$ confidence.

Bulk tank milk samples were collected during routine milk sampling by the Flemish milk control center (MCC-Vlaanderen) over the course of January 2016. All farms currently not enrolled in the milk quality control program of MCC were automatically excluded from our study. In 2016, 4,628 dairy herds were enrolled in the program, where 6,597 herds in total produced milk in Flanders (Flemish Department of Agriculture and Fisheries, 2016; MCC, 2016). Samples were cooled $\left(4-8^{\circ} \mathrm{C}\right)$ and immediately transported to the laboratory for analysis.

Commercially available real-time PCR (PathoProof Mastitis Complete 16 PCR assay, Thermo Fisher Scientific, Waltham, MA) and M. bovis antibody ELISA (BIO K 302, Bio-X Diagnostics S.A.) were performed on the samples according to the manufacturer's instructions (http://www.biox.com/en/bio-k-302-monoscreen -abelisa-mycoplasma-bovis-indirect-monowell-p-250/). Specifically for the ELISA, the manufacturers' recommended cutoff value of $37 \%$ optical density was used to guarantee the best combination of sensitivity and specificity. After determining the apparent prevalence, true prevalence was calculated using Epi Tools (Ausvet, 2018).

To collect information on potential risk factors, a questionnaire containing 44 questions related to internal and external biosecurity and general herd management was distributed to all farms by email (Table 1). The questionnaire was made available online through Google Forms (Alphabet Inc., Mountain View, CA). Nonresponders were interviewed by telephone. All interviews were done by the same researcher. Interviews were conducted in the second part of 2016. Further herd information (mean herd size, amount of purchase, mortality) was gathered from the national registry of cattle movements (SANITRACE). All questionnaire data were put into a spreadsheet (Microsoft Excel) and transferred to SAS 9.4. (SAS Institute Inc., Cary, Inc.) for statistical analysis.

To identify risk factors associated with the detection of M. bovis-specific DNA or antibodies in BTM, a multivariable logistic regression model was built (PROC LOGISTIC). The outcome variable was a PCR- or Ab ELISA-positive result. The elementary unit was the 
farm. In a first step, potential risk factors were tested univariably for their association with the outcome variable. After univariable analysis, variables with a $P$-value of 0.20 or less were withheld for multivariable analysis. Predictors were grouped and recoded if an insufficiently low number of cases was present in a given category. Several factors could not be included in the univariable analysis due to a too low number of observations. Correlation between different predictors was tested with Pearson's and Spearman rho correlation. If the correlation was above 0.6 , only the most significant variable was withheld for further analysis. Multivariable analysis was performed stepwise backward, gradually excluding nonsignificant variables $(P<0.05)$. Biologically relevant interactions between significant main factors were tested. Model fit was checked by the Hosmer-Lemeshow goodness of fit test. Significance was set at $P \leq 0.05$ and $P \leq 0.10$ was considered a trend.

\section{RESULTS}

\section{Prevalence and Herd Characteristics}

Overall, $7 \%(95 \% \mathrm{CI}=2.06-11.49 \%)$ of the farms tested positive by PCR on BTM, with a true prevalence of $7.1 \%$ (95\% CI $=2.06-11.49 \%$ ). Seventeen percent of all farms tested Ab ELISA-positive on BTM (95\% CI $=9.72-24.28 \%)$. The true prevalence was $24.8 \%(95 \%$ $\mathrm{CI}=16.42-33.15 \%)$ for the ELISA results. None of the farms that tested positive in PCR had detectable antibodies present in the BTM and none of the farms that were Ab ELISA BTM-positive were PCR-positive.

The response rate of the questionnaire was $55 \%$ $(55 / 100)$. Of these, 5 farms $(9 \%)$ were PCR-positive and 13 farms $(23,6 \%)$ were ELISA-positive. The mean herd size of the farms that filled in the questionnaire was 157 (range $=62-460$ animals), which was not significantly different from the target population (mean = 134) but did trend to be among the larger herds $(\mathrm{t}=$ $1.93 ; \mathrm{df}=53 ; P=0.06)$.

No milk from other herds was purchased and no bulls were shared with other herds by any of the 55 herds. Only 1 herd used a teaser bull for heat detection. None of the herds used automated milk feeders. All calves were housed inside after weaning. All of these factors were excluded from further analysis given the low number of observations in 1 or more categories.

Concerning biosecurity, only 1 of the herds applied hygienic measures to clean off premise vehicles. One fourth of all interviewed farmers indicated that they

Table 1. Questionnaire provided to the farmers to gauge the internal and external biosecurity and general herd management concerning Mycoplasma bovis: Overview on the data collected by questionnaire (all questions were yes vs. no questions unless otherwise specified between brackets)

\begin{tabular}{ll}
\hline Subject & Description \\
\hline M. bovis exposure & Previous positive tests on the farm; known colleagues with problems \\
Herd movements/exposure to other herds & $\begin{array}{l}\text { Trade show visits; calf rearing expedited; neighboring pastures with cows; use of farming } \\
\text { machines from other herds; use of farming equipment from other herds; trading of bulls } \\
\text { between farms; distance to nearest neighbor herd (in meters); months of pasture access for } \\
\text { young stock, heifers, cows; use of a purchase protocol (blood testing); use of a quarantine } \\
\text { period (yes, }>2 \text { wk/yes, maximum 1 wk/no) }\end{array}$
\end{tabular}

Environmental factors

Hygienic measures

Calf rearing and internal biosecurity
Drink water type (rainwater/city water/well water)

Herd visitors (farm-specific clothing/farm-specific boots/boot disinfectant/disinfection of car/none); bird control measures; rodent control measures; cleaning of calf pens (removal of straw and feces/high-pressure cleaning/disinfection); separate calving pen (yes, 1 cow at a time/yes, multiple animals at a time/no); use of calving pen by sick animals

Use of milk of another herd for calf rearing; use of colostrum of another herd; use of lyophilized colostrum; use of gamma-irradiated colostrum; calf disease representation in the last year (cough/head tilt/arthritis/umbilical infections/diarrhea/pneumonia/none of the above); colostrum providing (bottle/tube/suckling); individual housing (igloo inside/igloo outside/individual box in a separate young stock stable/individual box in adult stable); milk (cow milk/powder milk/suckling calves); age of grouping (in weeks); group housing (outside/box in young stock stable/box in stable of adult cows); use of an automatic milk feeder; calf contact when individually housed; individual drinking buckets; contact of calf caretaker with adult cows

Herd visitors Amount of visiting veterinarians; frequency of livestock-dealer visits (in weeks); visits by people also visiting veal farms

Reproduction Use of a breeding bull; use of a teaser bull 
Table 2. Results of univariable analysis of factors associated with a Mycoplasma bovis-positive test result on bulk tank milk (all variables with $P \leq 0.20)^{1}$

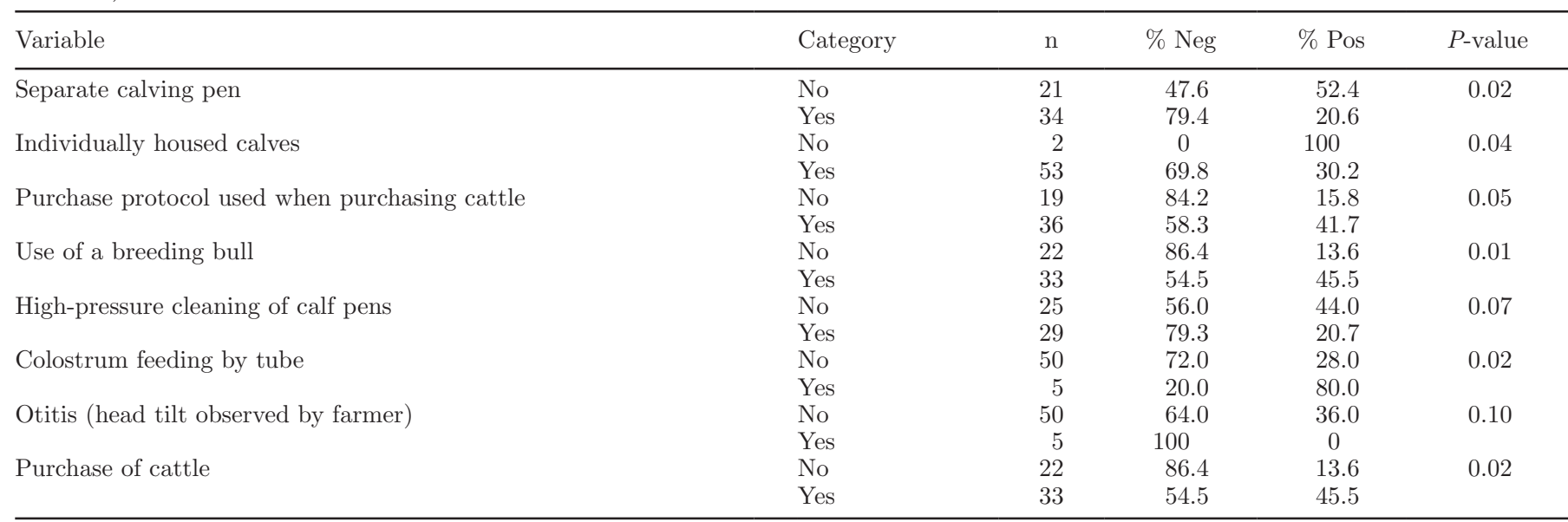

${ }^{1} \mathrm{Neg}=$ negative bulk tank milk sample; Pos $=$ PCR or antibody ELISA positive result.

did not enforce any hygienic measures for visitors at all. When farmers used a bull for some animals, but not the whole herd (e.g., to breed rebreeders), they were grouped in the "uses a bull for insemination" category. Sixty percent of the farms still used a bull on some or all cows.

\section{Risk Factor Analysis}

Due to the relatively small number of returned questionnaires, the outcome variable was adapted to "having a $M$. bovis-specific DNA- or antibody-positive BTM sample" instead of analyzing data from ELISA-positive herds separately from PCR-positive herds. After univariable analysis 8 variables with a $P$-value of 0.20 or less were withheld for multivariable analysis: the use of a separate calving pen, individual housing of calves, the use of a cattle purchase protocol (serological testing of a variety of diseases at purchase), the use of a breeding bull, high-pressure cleaning, colostrum feeding by tube, presence of otitis media, and purchase in the year before testing (Table 2). Purchase of cattle in the year before testing was significantly correlated with the presence of a bull, but the bull was more significantly linked to a positive sample. The final model consisted of 2 significant risk factors for a positive BTM result after multivariable analysis (Table 3). Farms which used a breeding bull had 4.7 higher odds to test positive. The use of a separate calving pen was a protective factor $($ odds ratio $=0.27)$.

\section{DISCUSSION}

We aimed to determine the prevalence of $M$. bovis in Belgium and to identify new risk factors. One hundred dairy farms were randomly selected, stratified on province.

Selection bias cannot be ruled out, as herds needed to participate in the milk control program to be eligible for our study. It is possible that these herds are, in general, larger and possibly more modern. In the present study, a relatively high response rate of the questionnaire of $55 \%$ was achieved. However, this still left the study with relatively low power due to the initial sample size, which could mean certain risk factors remain unidentified. As the questionnaire was made available online, and nonresponders were contacted by phone, a certain measure of observer bias was possible. However, to mitigate this, only 1 researcher conducted the interviews by telephone, following the same questionnaire as was made available online. Due to time constraints, interviews were performed more than 6 mo after the initial sampling. This might have been a cause of recall bias in the interviewees, although none indicated to have

Table 3. Final multivariable model for Mycoplasma bovis PCR- or antibody ELISA-positive bulk tank milk samples

\begin{tabular}{llcccc}
\hline Variable & Category & Herds $(\mathrm{n})$ & \% Positive & Odds ratio & 95\% CI \\
\hline Use of a breeding bull & No & 22 & 13.6 & Referent & \multirow{2}{*}{$1.1-19.8$} \\
\multirow{2}{*}{ Separate calving pen } & Yes & 33 & 45.5 & 4.7 & 0.04 \\
& No & 21 & 52.4 & Referent & $0.08-0.94$ \\
\hline
\end{tabular}


done a big shift in farming in the time passed. Only 1 sample per herd was taken, which could have affected the sensitivity of our study (Biddle et al., 2003).

In Belgium in 2009, $1.5 \%$ of all BTM samples from 3 consecutive samplings in 200 herds tested positive using bacterial culture (Passchyn et al., 2012). In our study, done on the same sample pool in $2016,7 \%$ of the tested herds had active circulation of $M$. bovis, detected through a PCR-positive BTM sample. This seems to be a notable increase especially because only 1 sample per herd was taken. However, comparing the 2 studies is difficult due to the difference in technique. When comparing to recent studies determining between-herd prevalence using PCR on BTM from the Netherlands (approximately 1\% in 2017; Hogenkamp, 2017), Denmark (1.6\% in 2015; Nielsen et al., 2015), and the southeast of France (0\% in 2011; Arcangioli et al., 2011), our findings seem to indicate a higher active infection rate in Belgian farms compared with other nearby countries. One possible explanation for this higher prevalence could be the intensive contact structure (40\% of all cattle born between 2005 and 2009 moved herds at least once; Ensoy et al., 2014) and high geographical density of Belgian herds. In contrast to PCR, Ab ELISA does not detect active circulation but can detect recent contact of a herd with $M$. bovis. The true prevalence using Ab ELISA on BTM in our study was $24.8 \%$. As this BTM ELISA technique is relatively new, only data of 1 other country, Denmark, is available to date. Nielsen et al. (2015) found a prevalence of $7.1 \%$ in BTM of Danish herds when using the same test with the recommended optical density cutoff percentage (ODC\%) of 37\%. Comparing BTM ELISA results with previous serological studies on $M$. bovis prevalence is difficult; Parker et al. (2017) found a relatively low correspondence between serological results and BTM ODC\%, suggesting a lower usability of the BTM test as a between-herd screening tool when trying to predict within-herd seroprevalence. Antibodies were detectable in the BTM for up to 12 mo after the initial outbreak. After this period, despite having further positive $M$. bovis culture or PCR results, the BTM ODC\% dropped under the detection limit. However, notwithstanding these limitations, in our opinion BTM analysis can still be a valid between-herd screening tool in the field (e.g., in a purchase protocol), especially when combined with PCR analysis, as it is practical and low cost. Previous research found virtually no overlap between PCR- or ELISA-positive BTM samples, except what could be explained by chance (Parker et al., 2017). This reflects our findings in the current study, where no overlap was seen between PCR and ELISA samples. A possible hypothesis of this lack of overlap is that, in newly infected herds, PCR-positive samples will appear at least 1 to 2 wk before seroconversion, by which time it can be expected that actively infected animals have shown signs of mastitis and are separated from the BTM (Nicholas et al., 2002). Accordingly, it is possible that M. bovis antibodies are developed in response to other $M$. bovisassociated diseases, such as pneumonia or arthritis, without subsequent shedding in milk. Regardless of the test, the results in our study seem to indicate a higher $M$. bovis prevalence level compared with neighboring countries.

One of the most interesting observations of our study was the association between the presence of a breeding bull and a M. bovis-positive BTM sample (DNA or Ab). Moreover, where previous purchase was identified as a risk factor for the presence of $M$. bovis in a herd (Burnens et al., 1999), we showed that, although purchase was part of the univariable risk factors, it was strongly associated with the presence of a breeding bull, and the breeding bull was the more significant factor of the 2 . Bulls are still often used in Belgian herds to breed with rebreeders and older cows (as a means to produce more valuable crossbreed calves). Next to the purchase of the bull, Belgian dairy herds are often closed, with yearround calving patterns. Herd size was not significantly linked to $M$. bovis presence on the farm in our study, in contrast with the findings of Thomas et al. (1981) and Fox et al. (2003) but consistent with the findings of Gonzalez et al. (1992). As the biggest herd included in our study only had around 460 animals, it is possible that the Belgian mean herd size is not big enough to be able to distinguish significant differences between smaller and larger herds. Another possibility is that purchase and herd size are linked as well, where larger herds might buy in (or have bought) cattle more often to sustain their growth.

In bulls, $M$. bovis colonizes the prepuce and the distal urethra (Fish et al., 1985), decreasing fertility (Bielanski et al., 2000). Seminal vesiculitis, epididymitis, and persistent infection along the seminal tract with concurrent shedding have been described (Kirkbride, 1987). Mycoplasma bovis has been found in vaginal swabs of apparently unaffected cattle and cattle suffering from clinical mastitis (Punyapornwithaya et al., 2010; Hazelton et al., 2018). It was isolated from uterine samples, out of aborted fetuses and in postsurgical seromas after caesarean section (Stalheim and Proctor, 1976; Pfützner and Sachse, 1996; Gille et al., 2016). When AI was performed with $M$. bovis-infected semen, persistent infection of the genital tract was seen, with shedding for up to 8 mo after AI (Hirth et al., 1966). The findings of the present study, suggesting the importance of the bull, combine well with a recent report of $M$. bovis introduction into 2 naïve herds in Finland by use of AI (Haapala et al., 2018). In the recent introduction 
of $M$. bovis in New Zealand, semen was suspected of being the source of introduction (Ministry for Primary Industries, 2017). Mycoplasma bovis in semen can survive cryopreservation for up to 18 mo (Hirth et al., 1966). The commonly used antimicrobial cocktail to decontaminate AI semen could be insufficient (Visser et al., 1999). Given recent observations and our current findings, it seems possible that the role of semen in the $M$. bovis epidemiology has been underestimated in the past.

A second remarkable observation was the protective nature of a separate calving pen for the $M$. bovis status of the herd. There seem to be 2 possible explanations to this protective effect. On one hand, it is possible that the periparturient immunity depression could induce shedding of higher numbers of $M$. bovis at calving. However, even though $M$. bovis has been isolated out of placenta and fetal fluids, no research has been done on the infectious capacity of $M$. bovis excreted during parturition (Stalheim and Proctor, 1976). On the other hand, the use of a calving pen might protect the immunosuppressed periparturient animals from infection by limiting contact with carriers present in the herd. Limiting contact between susceptible animals and shedders will in any case limit the exposure, with less shedding in the BTM concurrently. An interesting parallel can be drawn between $M$. bovis and Chlamydia sp., as both the breeding bull and calving away from the herd were shown to be risk factors for the latter as well (Kemmerling et al., 2009).

\section{CONCLUSIONS}

A considerable proportion of the Flemish dairy herds had recently been in contact with $M$. bovis at the time of this study. Based on PCR results, the active prevalence of $M$. bovis seems to be higher compared with studies in neighboring countries. This study identified having a breeding bull and the absence of the calving pen as risk factors for having an ELISA- or PCR-positive BTM sample. Further attention should be given to the role of the breeding bull and calving pen in the spread of $M$. bovis in a herd, as well as their potential role in the development of effective control and preventive measures for M. bovis.

\section{ACKNOWLEDGMENTS}

This research was financed by the Flemish cattle monitoring project ('Veepeiler Rund'), headed by the Flemish Animal Health Service (DGZ-Vlaanderen). The authors wish to thank the laboratory technicians and all participating farmers for their kind cooperation.

\section{REFERENCES}

Aebi, M., B. H. van den Borne, A. Raemy, A. Steiner, P. Pilo, and M. Bodmer. 2015. Mycoplasma bovis infections in Swiss dairy cattle: A clinical investigation. Acta Vet. Scand. 57:10.

Arcangioli, M. A., M. Chazel, E. Sellal, M. A. Botrel, P. Bezille, F. Poumarat, D. Calavas, and D. Le Grand. 2011. Prevalence of $M y-$ coplasma bovis udder infection in dairy cattle: Preliminary field investigation in southeast France. N. Z. Vet. J. 59:75-78.

Ausvet. 2018. Estimated true prevalence and predictive values from survey testing. Accessed Feb. 15, 2018. http://epitools.eu/content .php?page $=$ TruePrevalence.

Biddle, M. K., L. K. Fox, and D. D. Hancock. 2003. Patterns of mycoplasma shedding in the milk of dairy cows with intramammary mycoplasma infection. J. Am. Vet. Med. Assoc. 223:1163-1166.

Bielanski, A., J. Devenish, and B. Phipps-Todd. 2000. Effect of Mycoplasma bovis and Mycoplasma bovigenitalium in semen on fertilization and association with in vitro produced morula and blastocyst stage embryos. Theriogenology 53:1213-1223.

Burnens, A. P., P. Bonnemain, U. Bruderer, L. Schalch, L. Audige, D. Le Grand, F. Poumarat, and J. Nicolet. 1999. (The seroprevalence of Mycoplasma bovis in lactating cows in Switzerland, particularly in the republic and canton of Jura). Schweiz. Arch. Tierheilkd. 141:455-460.

Cai, H. Y., P. Bell-Rogers, L. Parker, and J. F. Prescott. 2005. Development of a real-time PCR for detection of Mycoplasma bovis in bovine milk and lung samples. J. Vet. Diagn. Invest. 17:537-545.

Calcutt, M. J., I. Lysnyansky, K. Sachse, L. K. Fox, R. A. J. Nicholas, and R. D. Ayling. 2018. Gap analysis of Mycoplasma bovis disease, diagnosis and control: An aid to identify future development requirements. Transbound. Emerg. Dis. 65:91-109.

Ensoy, C., C. Faes, S. Welby, Y. Van Der Stede, and M. Aerts. 2014. Exploring cattle movements in Belgium. Prev. Vet. Med. 116:89 101

Fish, N. A., S. Rosendal, and R. B. Miller. 1985. The distribution of Mycoplasmas and Ureaplasmas in the genital tract of normal artificial insemination bulls. Can. Vet. J. 26:13-15.

Flemish Department of Agriculture and Fisheries. 2016. Agriculture - Agricultural information of 2016. K. M. O. FOD Economie, Middenstand en Energie. Accessed Jan. 12, 2018. https://lv.vlaanderen .be/nl/voorlichting-info/feiten-cijfers/landbouwcijfers.

Fox, L. K., D. D. Hancock, A. Mickelson, A. Britten, and O. R. Kaaden. 2003. Bulk tank milk analysis: Factors associated with appearance of Mycoplasma sp. in milk. J. Vet. Med. B Infect. Dis. Vet. Public Health 50:235-240.

Fox, L. K., J. H. Kirk, and A. Britten. 2005. Mycoplasma mastitis: A Review of transmission and control. J. Vet. Med. B Infect. Dis. Vet. Public Health 52:153-160.

Gille, L., P. Pilo, B. R. Valgaeren, L. Van Driessche, H. Van Loo, M. Bodmer, S. Bürki, F. Boyen, F. Haesebrouck, P. Deprez, and B. Pardon. 2016. A new predilection site of Mycoplasma bovis: Postsurgical seromas in beef cattle. Vet. Microbiol. 186:67-70.

Godden, S., S. McMartin, J. Feirtag, J. Stabel, R. Bey, S. Goyal, L. Metzger, J. Fetrow, S. Wells, and H. Chester-Jones. 2006. Heattreatment of bovine colostrum. II: effects of heating duration on pathogen viability and immunoglobulin G. J. Dairy Sci. 89:34763483.

Gonzalez, R. N., P. M. Sears, R. A. Merrill, and G. L. Hayes. 1992 Mastitis due to Mycoplasma in the state of New York during the period 1972-1990. Cornell Vet. 82:29-40.

González, R. N., and D. J. Wilson. 2003. Mycoplasmal mastitis in dairy herds. Vet. Clin. North Am. Food Anim. Pract. 19:199-221.

Haapala, V., T. Pohjanvirta, N. Vähänikkilä, J. Halkilahti, H. Simonen, S. Pelkonen, T. Soveri, H. Simojoki, and T. Autio. 2018. Semen as a source of Mycoplasma bovis mastitis in dairy herds. Vet. Microbiol. 216:60-66.

Hazelton, M. S., P. A. Sheehy, K. L. Bosward, A. M. Parker, J. M. Morton, C. J. Dwyer, P. G. Niven, and J. K. House. 2018. Short communication: Shedding of Mycoplasma bovis and antibody responses in cows recently diagnosed with clinical infection. J. Dairy Sci. 101:584-589. 
Hirth, R. S., S. W. Nielsen, and W. N. Plastridge. 1966. Bovine salpingo-oophoritis produced with semen containing a Mycoplasma. Pathol. Vet. 3:616-632.

Hogenkamp, W. 2017. Mycoplasma in 1\% tankmelk aangetoond. in Boerderij. 2018. Accessed Feb. 7, 2018. https://www.boerderij .nl/Rundveehouderij/Nieuws/2017/7/Mycoplasma-in-1-tankmelk -aangetoond-154694E/.

Kemmerling, K., U. Muller, M. Mielenz, and H. Sauerwein. 2009. Chlamydophila species in dairy farms: Polymerase chain reaction prevalence, disease association, and risk factors identified in a crosssectional study in western Germany. J. Dairy Sci. 92:4347-4354.

Kirkbride, C. A. 1987. Mycoplasma, Ureaplasma, and Acholeplasma infections of bovine genitalia. Vet. Clin. North Am. Food Anim. Pract. 3:575-591.

Maunsell, F. P., and A. Donovan. 2009. Mycoplasma bovis infections in young calves. Vet. Clin. North Am. Food Anim. Pract. 25:139-177.

Maunsell, F. P., A. R. Woolums, D. Francoz, R. F. Rosenbusch, D. L. Step, D. J. Wilson, and E. D. Janzen. 2011. Mycoplasma bovis infections in cattle. J. Vet. Intern. Med. 25:772-783.

MCC. 2016. MCC Yearly Report 2016:14-15.

Ministry for Primary Industries. 2017. Analysis of risk pathways for the introduction of Mycoplasma bovis into New Zealand. Publications Logistics Officer Ministry for Primary Industries, Wellington, New Zealand.

Nicholas, R. A., and R. D. Ayling. 2003. Mycoplasma bovis: Disease, diagnosis, and control. Res. Vet. Sci. 74:105-112.

Nicholas, R. A., R. D. Ayling, and L. P. Stipkovits. 2002. An experimental vaccine for calf pneumonia caused by Mycoplasma bovis: Clinical, cultural, serological and pathological findings. Vaccine 20:3569-3575.

Nicholas, R. A., L. K. Fox, and I. Lysnyansky. 2016. Mycoplasma mastitis in cattle: To cull or not to cull. Vet. J. 216:142-147.

Nielsen, P. K., M. B. Petersen, L. R. Nielsen, T. Halasa, and N. Toft. 2015. Latent class analysis of bulk tank milk PCR and ELISA testing for herd level diagnosis of Mycoplasma bovis. Prev. Vet. Med. 121:338-342.

Parker, A. M., J. K. House, M. S. Hazelton, K. L. Bosward, J. M. Morton, and P. A. Sheehy. 2017. Bulk tank milk antibody ELISA as a biosecurity tool for detecting dairy herds with past exposure to Mycoplasma bovis. J. Dairy Sci. 100:8296-8309.

Passchyn, P., S. Piepers, L. De Meulemeester, F. Boyen, F. Haesebrouck, and S. De Vliegher. 2012. Between-herd prevalence of $M y-$ coplasma bovis in bulk milk in Flanders, Belgium. Res. Vet. Sci. 92:219-220.

Perez-Casal, J., T. Prysliak, T. Maina, M. Suleman, and S. Jimbo. 2017. Status of the development of a vaccine against Mycoplasma bovis. Vaccine 35:2902-2907.

Petersen, M. B., K. Krogh, and L. R. Nielsen. 2016. Factors associated with variation in bulk tank milk Mycoplasma bovis antibodyELISA results in dairy herds. J. Dairy Sci. 99:3815-3823.

Pfützner, H., and K. Sachse. 1996. Mycoplasma bovis as an agent of mastitis, pneumonia, arthritis and genital disorders in cattle. Rev. Sci. Tech. 15:1477-1494.

Punyapornwithaya, V., L. K. Fox, D. D. Hancock, J. M. Gay, and J. R. Alldredge. 2010. Association between an outbreak strain causing Mycoplasma bovis mastitis and its asymptomatic carriage in the herd: a case study from Idaho, USA. Prev. Vet. Med. 93:66-70.

Sachse, K., H. Pfutzner, H. Hotzel, B. Demuth, M. Heller, and E. Berthold. 1993. Comparison of various diagnostic methods for the detection of Mycoplasma bovis. Rev. Sci. Tech. 12:571-580.

Sachse, K., H. S. Salam, R. Diller, E. Schubert, B. Hoffmann, and H. Hotzel. 2010. Use of a novel real-time PCR technique to monitor and quantitate Mycoplasma bovis infection in cattle herds with mastitis and respiratory disease. Vet. J. 186:299-303.

Stalheim, O. H., and S. J. Proctor. 1976. Experimentally induced bovine abortion with Mycoplasma agalactiae subsp bovis. Am. J. Vet. Res. 37:879-883.

Thomas, C. B., P. Willeberg, and D. E. Jasper. 1981. Case-control study of bovine mycoplasmal mastitis in California. Am. J. Vet. Res. 42:511-515.

Visser, I. J., E. A. ter Laak, and H. B. Jansen. 1999. Failure of antibiotics gentamycin, tylosin, lincomycin and spectinomycin to eliminate Mycoplasma bovis in artificially infected frozen bovine semen. Theriogenology 51:689-697. 